\title{
APLICAÇÃO DO SIMULADOR EMSO EM UM PROBLEMA ESPECÍFICO DE CINÉTICA E CÁLCULO DE REATORES
}

\author{
T. A. F. ROCHA ${ }^{1}$, W. U. LEITE ${ }^{1}$, B. L. VERÁS ${ }^{1}$ e W. R. O. PIMENTEL ${ }^{1}$ \\ ${ }^{1}$ Universidade Federal de Alagoas, Centro de Tecnologia \\ E-mail para contato: wildson_leite@hotmail.com
}

\begin{abstract}
RESUMO - O presente trabalho visa mostrar como, utilizando o programa EMSO (Environment for Modeling, Simulationand Optimization), podem-se solucionar problemas que envolvem os principais reatores cinéticos: o reator batelada, o reator de mistura perfeita (CSTR - Continuous Stirred - Tank Reactor) e reator tubular (PFR - Plug Flow Reactor). Aqui é mostrado um problema específico, envolvendo os três reatores.
\end{abstract}

\section{INTRODUÇÃO}

A modelagem e simulação são fundamentais quando se deseja fazer previsões sobre o comportamento de um processo químico em diferentes condições de operação. Em modelos matemáticos podemos descrever quantitativamente os processos, os parâmetros podem ser variados facilmente em grandes intervalos, ao contrário de investigações experimentais, que podem ser caras e longas (SCHULTZ et al., 2013).

Quando se objetiva estudar o comportamento das variáveis de um processo no decorrer do tempo, o uso de simuladores torna-se uma ferramenta facilitadora e eficaz. Nesse, sentido, utilizamos o EMSO que é um simulador dinâmico baseado em equações para tal finalidade, e este não apresenta custos de utilização. Nele é possível fazer modelagens e simulações de processos simples ou complexos, no modo estacionário ou dinâmico. Onde o usuário pode criar seus próprios modelos utilizando a linguagem de modelagem do próprio EMSO(MANUAL DE TREINAMENTO DO SIMULADOR EMSO, 2010).

Neste estudo trataremos dos três principais reatores abordados no ensino de cinética e cálculo de reatores, o primeiro é o reator batelada, que é um reator simples e requer poucos acessórios. É ideal para a produção em pequena escala, pode ser empregado para experimentos de cinética das reações, para a fabricação de produtos caros e nos processos difíceis de ser convertido em operações contínuas. Como em todo processo descontínuo e semi-contínuo, o reator batelada opera em regime transiente por natureza. Para fins de projeto, assume-se possuir agitação perfeita, implicando não haver variação espacial na velocidade de ração. O segundo é o PFR que opera continuamente e em estado estacionário, por isso, é ideal para produção em larga escala quando a taxa de reação é razoavelmente ou extremamente alta. Este reator é modelado em função de uma diferencial de volume. Por fim, o CSTR.Este é um reator agitado que opera continuamente em estado estacionário, seu conteúdo é considerado perfeitamente misturado. Consequentemente, entende-se que a temperatura, a concentração e a velocidade da reação dentro de um CSTR independem do tempo, quando atingido o regime estacionário, ou da posição espacial das partículas da mistura (FOGLER, 2009). 
Temos por objetivo, a partir da problemática descrita na metodologia a seguir, elaborar a modelagem e a simulação dos três reatores já mencionados e, através dos gráficos que descrevem o comportamento dos mesmos, demonstrar que o simulador EMSO é eficaz na resolução de problemas dessa natureza.

\section{METODOLOGIA}

Rodrigues et al. (2006), afirma que o livro-texto "Elementos de Engenharia das Reações Químicas" de Fogler(2009) tem se consolidado como a principal literatura no ensino de cinética e cálculo de reatores. Ele acredita que a abordagem dos exemplos e dos problemas propostos, que muitas vezes requer o uso de ferramentas computacionais, seja o motivo para o sucesso desse livro.

Adaptando o Problema 4-5, proposto por Fogler (2009), temos que escolher entre um reator CSTR e um PFR, aquele que propicie a maior conversão $X$ de reagente em produto. $\mathrm{O}$ CSTR tem 200,0 L de volume operando isotermicamente, podendo ser resfriado a $0^{\circ} \mathrm{C}$ ou aquecido a $77^{\circ} \mathrm{C}$. O reator PFR tem $800,0 \mathrm{~L}$ de volume, também opera isotermicamente, mas somente a temperatura de $27^{\circ} \mathrm{C}$. Posteriormente, o problema propõe que o tempo de reação seja calculado, caso ela ocorra num reator batelada de 200,0 L de volume, em duas condições, a $0^{\circ} \mathrm{C}$ e a $77^{\circ} \mathrm{C}$.

A reação proposta é genérica, ocorre isotermicamente em fase líquida e segue uma lei elementar de velocidade.Ela tem a forma:

$A+B \rightarrow C$

Onde $A$ e $B$ são reagentes, ambos de concentração de alimentação de $2 \mathrm{~mol} / \mathrm{L}$ antes da mistura. A vazão volumétrica de cada corrente é de $5 \mathrm{~L} / \mathrm{min}$ e suas temperaturas na entrada é de $300 \mathrm{~K}$. As correntes de reagentes são misturadas imediatamente antes de entrarem no reator. São fornecidas ainda a velocidade específica $k$ e a energia de ativação $E$ dessa reação, a saber, $0,07 \mathrm{~L} / \mathrm{mol} / \mathrm{min}$ a $300 \mathrm{~K}$ e $20000 \mathrm{cal} / \mathrm{mol}$, respectivamente.

Para melhor compreensão, a Figura 1 mostra o esquema simplificado do processo.

Figura 1 - Esquema do processo de reação.

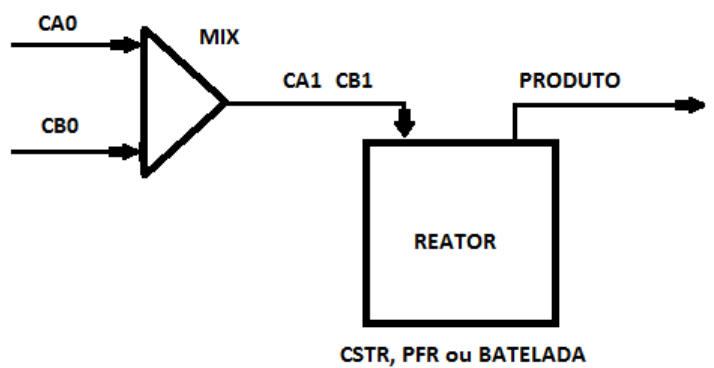

Visto que as correntes de reagentes são misturadas imediatamente antes de entrar no reator, as concentrações das espécies $A$ e $B$ são divididas pelo número de correntes misturadas. Como nesse caso duas correntes são misturadas, as novas concentrações dos reagentes foram reduzidas à metade, conforme a Equação 1, abaixo. 


$$
C_{i 1}=\frac{C_{i 0}}{2}
$$

Ainda devido a mistura, as vazões mássicas $v$ das correntes líquidas são somadas, conforme Equação 2.

$$
\mathrm{v}=\mathrm{v}_{A 0}+\mathrm{v}_{B 0}
$$

Assumindo que a espécie $A$ seja o reagente limitante e que os reatores tenham volume constante, a estequiometria em termos da conversão, assumirá a forma da Equação 3 e a Equação 4.

$$
\begin{aligned}
& C_{A}=C_{A 1}\left(1-X_{A}\right) \\
& C_{B}=C_{A 1}\left(\frac{C_{B 1}}{C_{A 1}}-\beta X_{A}\right)
\end{aligned}
$$

Onde $\beta$ é o coeficiente estequiométrico do reagente $B$, neste caso, igual a 1 . Como consequência da alimentação de concentrações equivalentes das espécies $A$ e $B$, temos que a Equações 3 e 4, assumirá a forma da Equação 5 abaixo.

$$
C_{B}=C_{A 1}\left(1-X_{A}\right)
$$

Assim, temos que as concentrações finais dos reagentes são iguais $\left(C_{A}=C_{B}\right)$.

Sabendo que a reação obedece a uma lei elementar de velocidade, temos que a velocidade de consumo de $A,-r_{A}$, será dada pela Equação 6 .

$$
-r_{A}=k C_{A} C_{B}=k C_{A 1}^{2}\left(1-X_{A}\right)^{2}
$$

Por Arrhenius, a velocidade específica $k$ de reação, é função exclusiva da temperatura. Assim sendo, fazemos uso equação 7 proposta por Arrhenius.

$$
k_{2}=k_{1} \exp \left[\frac{E}{R}\left(\frac{1}{T_{1}}-\frac{1}{T_{2}}\right)\right]
$$

Onde $R$ é a constante universal dos gases e tem valor igual a $1,987 \mathrm{cal} / \mathrm{mol} / \mathrm{K}, k_{l}$ é a velocidade específica da reação na temperatura absoluta $T_{1}(0,07 \mathrm{~L} / \mathrm{mol} / \mathrm{min}$ a $300 \mathrm{~K})$ e $k_{2}$ é a velocidade específica da reação na temperatura absoluta desejada $T_{2}$.

Sendo assim, os três reatores de interesse, foram modelados pelas Equações 8, 9 e 10, que se seguem. Vale ressaltar que a condição simplificadora que fala a respeito da operação em estado estacionário do reator CSTR, não foi assumida, com a finalidade de demonstrar a desempenho do simulador EMSO na resolução de equações diferenciais ordinárias. Esta hipótese foi aplicada somente ao reator PFR.

Assim, o CSTR foi modelado pela Equação 8,

$\frac{d X_{A}}{d t}=k_{2} C_{A 1}\left(1-X_{A}\right)^{2}-\frac{v X_{A}}{V}$

O PFR pela Equação 9.

(9)

$$
\frac{X_{A}}{1-X_{A}}=\frac{V k_{1} C_{A 1}}{\mathrm{v}}
$$

O reator Batelada pela Equação 10.

$\frac{d X_{A}}{d t}=k_{2} C_{A 1}\left(1-X_{A}\right)^{2}$

Nas três equações acima, $V$ representa o volume do respectivo reator.

Após esta etapa, os modelos foram implementado no simulador EMSO, conforme Figura 2, abaixo. 
Figura 2 - Implementação dos modelos no simulador EMSO.

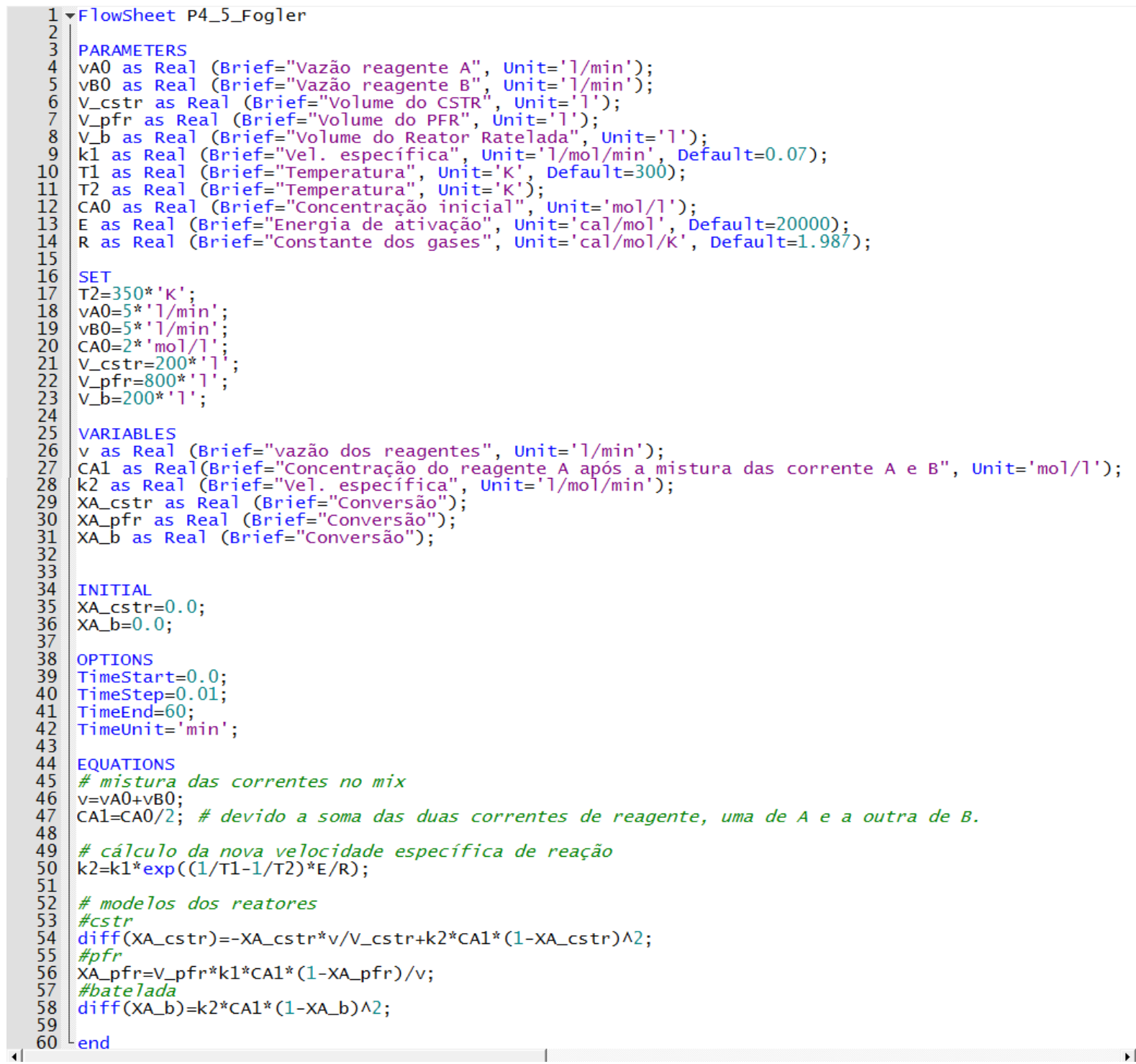

$\mathrm{Na}$ entidade do EMSO, denominada FlowSheet, os parâmetros foram declaradas na seção PARAMETRS, as variáveis em VARIABLES, as condições iniciais em INITIAL e as equações em EQUATIONS. A seção $S E T$ foi implementada a fim de facilitar a alteração dos valores parâmetros e a seção INITIAL foi colocada, pois é imprescindível em sistemas dinâmicos, ou seja, cujas variáveis se alterem em função do tempo.

\section{RESULTADOS}

Como pode ser observado no gráfico da Figura 3 , o CSTR operando a $0^{\circ} \mathrm{C}(227 \mathrm{~K})$ apresenta conversão inexpressiva de reagente. Já operando a $77^{\circ} \mathrm{C}(350 \mathrm{~K})$, o mesmo chega à conversão de $A$ próxima a $0,90(90 \%)$ quando o regime estacionário é atingido. Nessa temperatura, o regime é atingido por volta dos $200 \mathrm{~s}$ (3,3 min). A $300 \mathrm{~K}$ o PFR teve conversão próxima a $0,85(85 \%)$. Vale ressaltar que os reatores operam isotermicamente e que o PFR 
não foi modelado para descrever o seu regime dinâmico, isso explica a reta gerada para a conversão obtida por este reator.

Figura 3 - Conversão do reagente $A$ em função do tempo, para os reatores CSTR e PFR em diferentes temperaturas de operação.

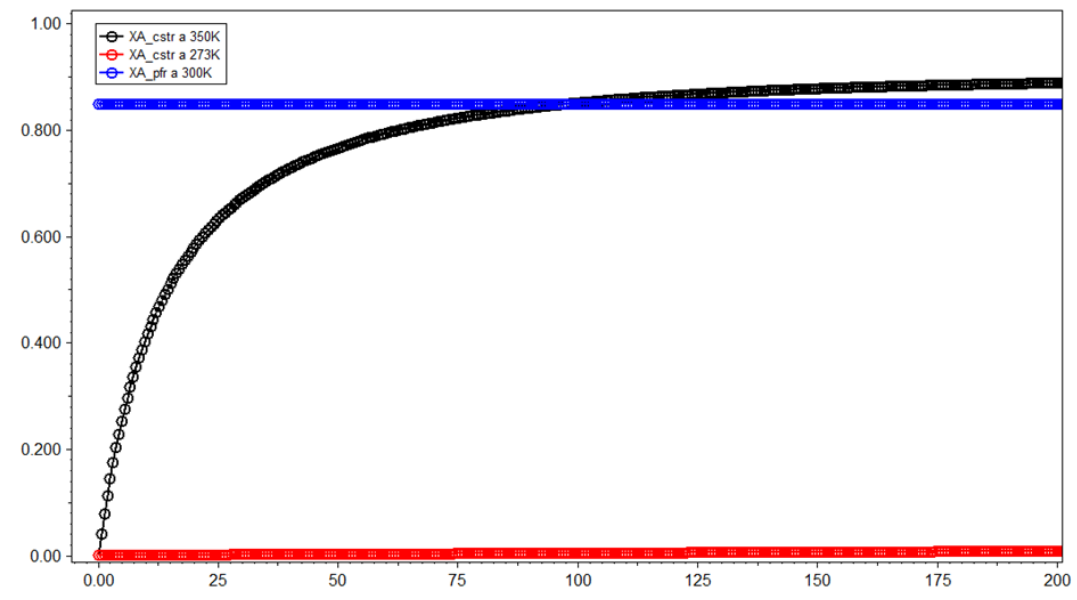

Assim, analisando a conversão de reagente em produto, o CSTR operando a $77^{\circ} \mathrm{C}$ $(350 \mathrm{~K})$ é o melhor reator para a reação proposta. Vale ressaltar também, que o CSTR apresentou uma maior conversão com apenas um quarto do volume do CSTR, isso é outra vantagem a ser considerada.

Fazendo a análise da conversão em função do tempo para a reação ocorrendo isotermicamente a $77^{\circ} \mathrm{C}$ num reator batelada, obtivemos o gráfico da Figura 4. Nessa figura, somos capazes de observar que o tempo para a conversão de 0,90 (90\%) foi de aproximadamente 60s $(1 \mathrm{~min})$. Com o intuito de observar o tempo para esta mesma conversão, neste mesmo reator, só que operando a $0^{\circ} \mathrm{C}(227 \mathrm{~K})$. Neste último gráfico, somos capazes de observar que foram necessários aproximadamente $2,13 \times 10^{5} \mathrm{~s}$ ( 2,5 dias), para se atingir $90 \%$ de conversão.

Figura 4 - Conversão do reagente $A$ em função do tempo num reator batelada a 350K.

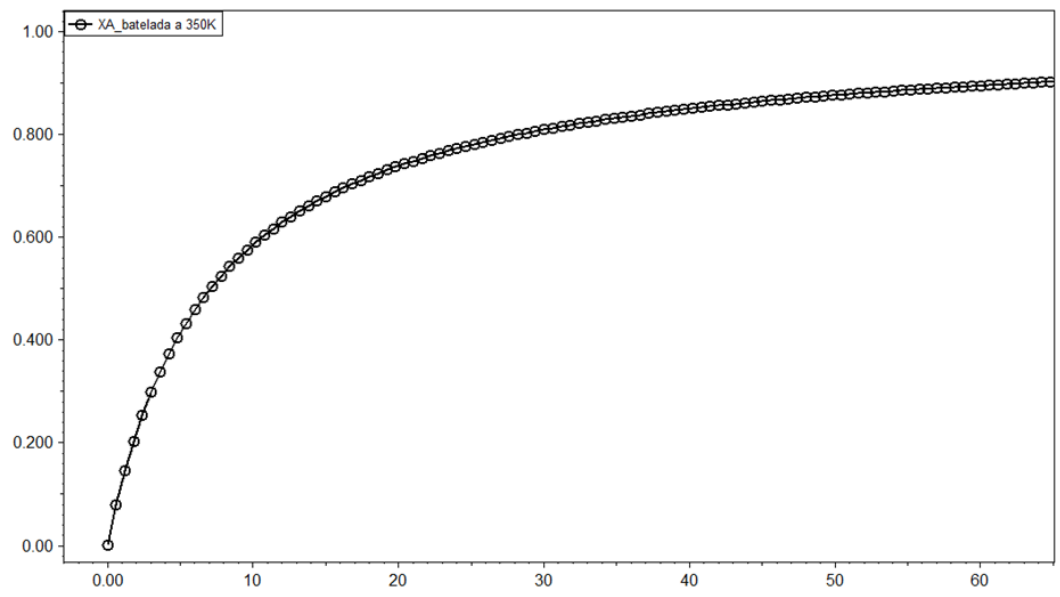


Figura 5 - Conversão do reagente $A$ em função do tempo num reator batelada a $273 \mathrm{~K}$.

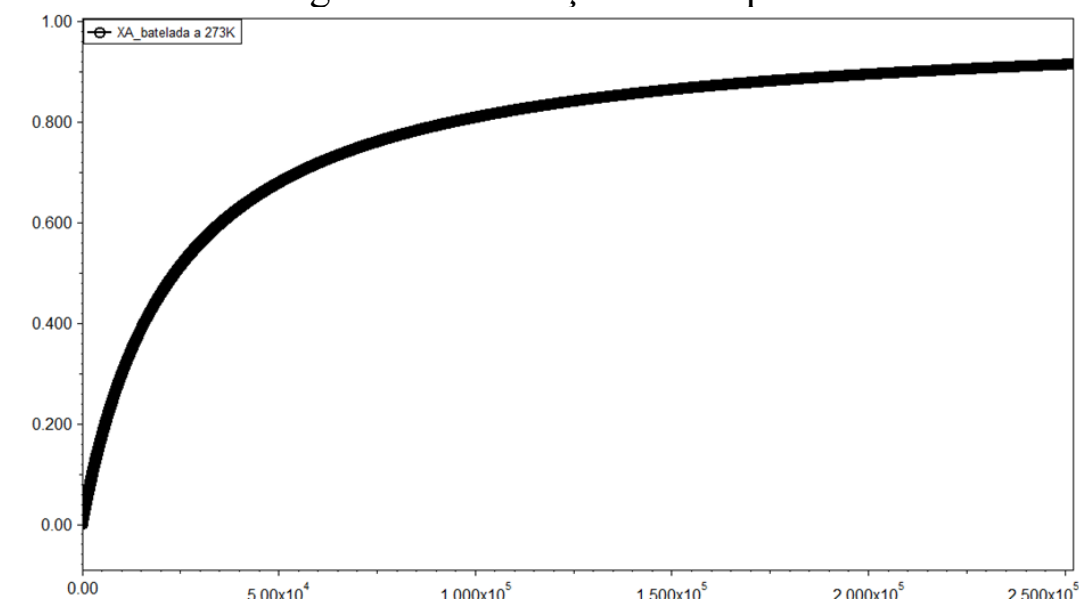

\section{CONCLUSÃO}

Com este trabalho conseguimos demonstrar que o simulador EMSO é eficiente e prático na resolução de problemas acadêmicos de cinética e cálculo de reatores. Constatamos ainda que os resultados obtidos são compatíveis aos apresentados por Fogler (2009) e condizentes com a literatura. E levando em consideração que o presente problema, temos que o CSTR a $350 \mathrm{~K}$ apresenta maior conversão quando comparado ao CSTR a $273 \mathrm{~K}$ e ao PFR a $300 \mathrm{~K}$, contudo a melhor conversão por tempo se dá no reator batelada a $350 \mathrm{~K}$.

\section{REFERÊNCIAS}

FOGLER, H. S. Elementos de engenharia das reações químicas. $4^{\mathrm{a}}$ ed. Rio de Janeiro, LTC, 2009.

SCHULTZ, G; et al. Simulação do reator químico de retromistura no software EMSO.Aracruz, 2013.

RODRIGUES, R; et al. XVI Congresso Brasileiro de Engenharia Química, 2006, Rio Grande do Sul. Ensino de cinética e cálculo de reatores químicos utilizando o simulador EMSO.

Porto Alegre; UFRGS, 2006.

Manual de treinamento do simulador EMSO, 2010. 